\title{
Experimental Investigations of Forerunners at the Fronts of Localized Disturbances of a Straight Wing Boundary Layer
}

\author{
V. N. Gorev, M. M. Katasonov*, V. V. Kozlov \\ Khristianovich Institute of Theoretical and Applied Mechanics, Novosibirsk 630090, Russia \\ Email:mikhail@itam.nsc.ru
}

\begin{abstract}
In recent years the longitudinal localized vortex disturbances, so called "streaky structures", or "puffs" appearing in boundary layers under the effect of external flow turbulence are of much interest. Once these disturbances are generated, they amplify downstream, turn into the incipient spots, and finally, result in laminar-turbulent transition " 1,2 ". In the present work wave packets (forerunners) occurring in the regions preceding a drastic change of flow velocity inside the boundary layer at the localized disturbances fronts are in focus. Their characteristics and dynamics have been studied experimentally in straight wing boundary layer. The investigations were carried out in the subsonic low-turbulent wind tunnel T-324, ITAM SB RAS. Free stream velocity was in the range $4<U_{\infty}<8 \mathrm{~m} / \mathrm{s}$. Free stream turbulence level was in the range $0.04 \% U_{\infty}<T u<0.79 \% U_{\infty}$. The high level of free stream turbulence was generated from grid located at the beginning of the wing tunnel test section. One model of a straight wing was $1000 \mathrm{~mm}$ span with the chord of $450 \mathrm{~mm}$, an other model was 200 $\mathrm{mm}$ span and $290 \mathrm{~mm}$ chord. The models were set vertically at positive $\left(5^{\circ}\right)$ and zero angle of attack. The localized disturbances (streaks) were generated via blowing (suction) through a thin slot arranged in the surface parallel to the leading edge. In the present experiments the high-frequency perturbations, i.e. forerunners, at the leading and back fronts of the localized disturbances evolving in the boundary layer have been found. Their characteristics affected by the external-flow pressure gradient, free stream turbulence level $(T u)$, the way of the longitudinal structures generation, and velocity gradients induced by the latter were investigated. In particular, it was observed that the forerunners' are strongly amplified in the adverse pressure gradient flow being much influenced by local velocity gradients. The results of the study make reason to consider the forerunners as wave packets of 3D instability waves. It was found that at downstream development of the forerunners they transform into the $\Lambda$-structures.
\end{abstract}

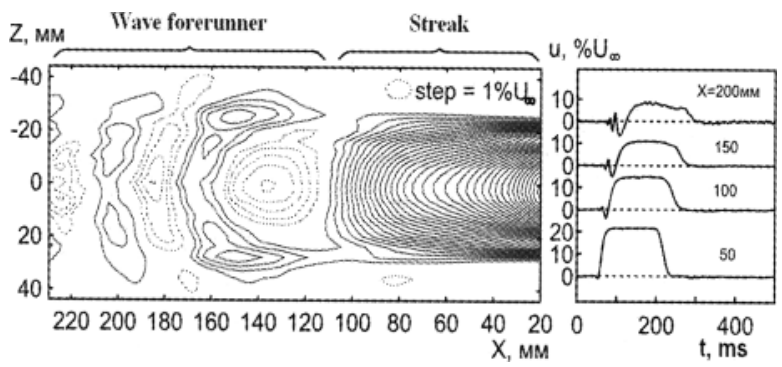

Figure: Hot-wire anemometry visualization of the streak and the wave forerunner at the leading edge of the streak. The streak was generated by suction through the spanwise slot

\section{REFERENCES}

1. Kozlov. Euromech Colloquim 353: Booklet of summaries. Karlsruhe, 1996

2. Boiko, et al. Berlin; Heidelberg; New York: Springer Verlag, 2002 